
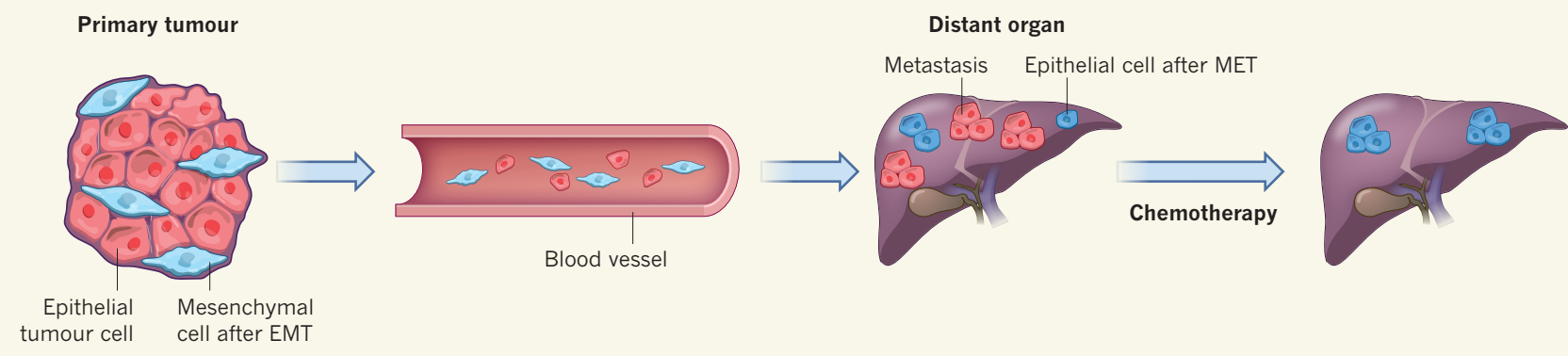

Figure 1 | Metastatic potential. A small fraction of epithelial cells in a solid tumour acquires mesenchymal-cell characteristics during tumour progression, through a process known as epithelial-to-mesenchymal transition (EMT). Both epithelial and mesenchymal cancer cells can invade the bloodstream and exit it at distant sites, where the mesenchymal cells undergo a reverse mesenchymal-to-epithelial (MET) transition. Contrary to previous opinion, Fischer et al. ${ }^{3}$ and Zheng et al. ${ }^{4}$ find that the majority of metastatic tumours at secondary sites are initiated by epithelial cells from the primary tumour, and not by cells that have undergone EMT and subsequent MET. However, both research groups also show that such transitioned cells are more resistant to chemotherapeutic drugs than are untransitioned epithelial cells and emerge as the dominant metastatic population following treatment.
EMT-switched tumour cells that had reached the lung. Analysis of the genes being expressed in these cells correlated this resistance with increased expression of genes encoding drugmetabolizing enzymes and drug-transporter proteins. These findings were mirrored in Zheng and colleagues' Snail- or Twist-deleted pancreatic cancers, in which tumour cells with epithelial characteristics expressed higher levels of nucleoside-transporter proteins than did mesenchymal cells, potentially rendering the epithelial cells more sensitive to the chemotherapeutic drug gemcitabine.

These findings challenge the prevailing hypothesis that EMT is a key element in the metastatic dissemination of epithelial cancers, and they point to a distinct role of this cell-fate transition in enhancing cancer-cell survival during drug treatment. How can these data be reconciled with compelling previous reports on the role of EMT in metastasis? The earlier work studied the effects of EMT induced by the growth factor TGF $\beta$ or by overexpression of transcriptional regulators such as Snail or Twist. Such approaches might not capture the physiological process that occurs spontaneously in cancer cells as accurately as do the methods used in the current studies. It is probable that the induction of EMT that occurs during the natural progression of a cancer may be more subtle than the full EMT switch that is induced by the expression of powerful regulators and that has been associated with high levels of metastasis.

However, the limitations of the models used by Fischer et al. and Zheng et al. need to be considered before EMT-mediated tumour invasion can be dismissed outright. EMT is orchestrated by complex circuitry involving multiple signalling molecules and transcription factors. Tracing switched cells on the basis of expression of a single gene may therefore not fully capture these complicated features. Similarly, Snail and Twist function redundantly in many settings ${ }^{2,7,8}$, and inactivation of both (and potentially of other transcriptional regulators) simultaneously, rather than individually, may be required to abrogate EMT. Furthermore, cancer is a highly variable disease, and its full complexity cannot be completely captured in mouse models that are driven by expression of a few cancer-initiating genes. Nonetheless, the conclusions reached by the two studies warrant a re-evaluation of the role of EMT in cancer progression. Alternative ways in which epithelial cells could enter the bloodstream without acquiring mesenchymal properties, such as collective epithelial-cell migration ${ }^{9}$ or tumour fragmentation $^{10}$, are worth investigating.

The postulated role of EMT in mediating cancer-cell survival is reinforced by the two latest studies. Indeed, EMT has been linked to drug susceptibility of cancer cells, as well as to their entrance into a non-proliferative state in which they have stem-cell-like properties ${ }^{11}$. Understanding the many cellular pathways that together determine these cell fates, and how these pathways are modulated, is likely to provide fertile ground for drug discovery and for new therapeutic strategies.
Shyamala Maheswaran and Daniel A. Haber are at the Massachusetts General Hospital Cancer Center, Harvard Medical School, Boston, Massachusetts 02114, USA. e-mails:maheswaran@helix.mgh.harvard.edu; dhaber@mgh.harvard.edu

1. Thiery, J. P., Acloque, H., Huang, R. Y. \& Nieto, M. A. Cell 139, 871-890 (2009)

2. Ye, X.\& Weinberg, R. A. Trends Cell Biol. 25, 675-686 (2015).

3. Fischer, K. R. et al. Nature 527, 472-476 (2015).

4. Zheng, X. et al. Nature 527, 525-530 (2015).

5. Yu, M. et al. Science 339, 580-584 (2013).

6. Singh, A. \& Settleman, J. E Oncogene 29, 4741-4751 (2010).

7. Peinado, H., Olmeda, D. \& Cano, A. Nature Rev Cancer 7, 415-428 (2007).

8. Puisieux, A., Brabletz, T. \& Caramel, J. Nature Cell Biol. 16, 488-494 (2014).

9. Clark, A. G. \& Vignjevic, D. M. Curr. Opin. Cell Biol. 36, 13-22 (2015)

10.Aceto, N. et al. Cell 158, 1110-1122 (2014).

11.Rumman, M., Dhawan, J. \& Kassem, M. Stem Cells 33, 2903-2912 (2015).

This article was published online on 11 November 2015.

\title{
Hidden reservoirs
}

West Africa's Ebola epidemic continues to reveal surprises. Although the animal species that originally passed the virus to people remains a mystery, a virus reservoir and persistent disease have been identified in some human survivors.

\section{JONATHAN L. HEENEY}

A nimals are reservoirs for many pathogens that occasionally jump species and infect humans. In December 2013 in the forests of Guinea, a two-year-old boy became infected with the Zaire strain of Ebola virus from an unidentified animal source ${ }^{1}$. This event triggered the largest and longest human epidemic of Ebola viral infection in recorded history. Across several countries in
West Africa, over 28,000 people were infected and more than 11,000 died. This fatality rate of less than $50 \%$ was lower than in most previous outbreaks, and it left more than 16,000 survivors ${ }^{2}$. Studies of these survivors are changing our understanding of Ebola virus infection and raising concern for the long-term well-being of these individuals and their communities. Writing in the New England Journal of Medicine, Deen et al. ${ }^{3}$ reveal that Ebola virus RNA can persist in the semen of men for months 


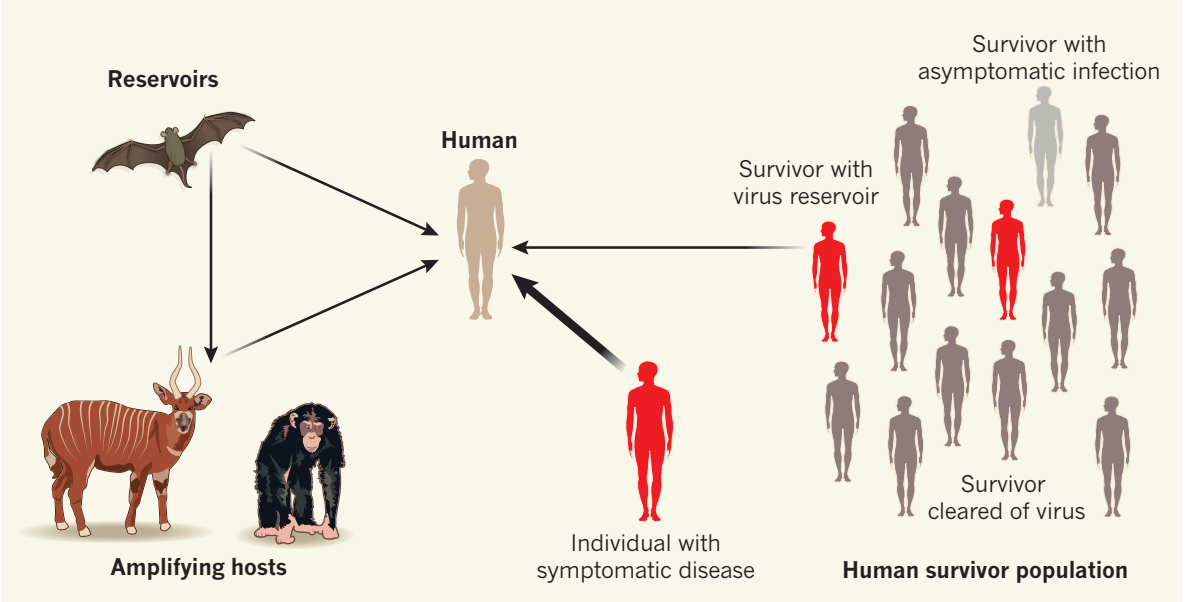

Figure 1 | Ebola infection dynamics in animals and humans. Ebola virus has been identified in several animal species, including bats, chimpanzees and forest antelopes. Transmission to humans can occur directly from reservoir species, in which the virus may persist without causing active infection, or from amplifying host species, in which the virus replicates to high levels, often causing illness and death. Most infected people develop acute Ebola virus disease and are highly infectious, although some individuals survive exposure and infection without developing symptoms. There is also growing evidence ${ }^{3,4}$ that the virus can persist in the central nervous system and reproductive organs of some survivors of the disease, with the possibility that these survivors could infect others months after resolution of their acute symptoms ${ }^{4}$.

after their recovery from the disease, and Mate et al. ${ }^{4}$ demonstrate that such persistence can be the source of new infections through sexual transmission.

Deen and colleagues obtained semen samples from 93 Sierra Leonian men who had survived Ebola virus disease (EVD) at various intervals after the onset of their disease. Although the authors find that the proportion of men whose semen contained Ebola virus RNA waned with time, the viral genomes persisted for as long as 7-9 months after recovery. Mate and colleagues provide convincing evidence that a female patient in Liberia, who subsequently died, had contracted Ebola virus through unprotected vaginal intercourse with her male partner, who had survived EVD.

These observations support similar findings in previous epidemics of filoviruses, the virus family to which Ebola belongs. There have been reports ${ }^{5,6}$ of the persistence of Marburg virus in the anterior chamber of the eye and semen of human survivors, and of the persistence of Ebola virus in the semen of men who survived the 1995 outbreak in the Democratic Republic of the Congo ${ }^{7}$. This has obvious implications for sexual partners.

The fact that Ebola virus is found at high levels in placental tissues also suggests that transmission could occur from pregnant women who survive EVD to their babies, although pregnant women who become infected usually abort the fetus before term ${ }^{8}$. Mother-to-child transmission by breastfeeding in survivors of Marburg virus has been reported ${ }^{9}$, and the potential for transmission through breast milk has also been suggested for Ebola ${ }^{10}$.

Although the relative risk of virus transmission from survivors is low compared with transmission from patients with acute EVD, a single case of new infection is sufficient to trigger an epidemic (Fig. 1). Thus, there is a strong need for rigorous assessment of the tissue reservoirs of Ebola in human survivors and the associated public-health risks. Follow-up health care should be combined with compassionate education of survivors and their communities by qualified and knowledgeable personnel, including advice on condom use.

Another lesson to emerge from this epidemic is that some survivors experience symptoms after their recovery from the main disease episode, suggesting that viral persistence in certain compartments of the body is more serious in some survivors than previously recognized. Reported symptoms include blurred vision, pain behind the eyes, hearing deficits, painful swallowing, joint pain, fever, memory loss and difficulty in sleeping ${ }^{11,12}$. The rehospitalization of a British nurse who developed neurological complications more than 9 months after surviving acute $\mathrm{EVD}^{13}$ is a chilling indication that the virus can persist in the central nervous system and be triggered to reactivate or to escape immune surveillance, or both. Fortunately, diagnosis and successful clinical intervention were possible for the nurse in Britain, but this situation is unlikely in most communities in West Africa.

The existence of a reservoir state in human Ebola survivors is now beyond debate. But we do not know how long viable virus can persist in these tissue reservoirs, nor whether the virus replicates there at low levels or is dormant and then triggered to replicate. Better definition and understanding of the reservoirs and the underlying mechanisms of post-EVD symptoms are needed to inform clinical management and treatment.

For example, studies of survivors may identify features of their immune responses (such as neutralizing-antibody determinants) that correlate with either full viral clearance or the persistence of viral reservoirs. Such correlates may enable survivors to be classified into 'carrier' or 'cleared' subtypes. Potential factors that could predispose survivors to viral reemergence also need to be taken into account, including genetics, compromised immunity owing to poor health, concurrent infections such as HIV, or use of immunosuppressive drugs. However, Ebola, like other RNA viruses, may be prone to mutational changes, and virus escape from the host's immune response may eventually occur even without predisposing factors.

It is also not clear how, or whether, postEVD immunity is affected by the stage of treatment or type of therapy given, such as monoclonal antibodies or the antibodies in convalescent plasma. As well as helping to classify survivors, enhanced understanding of viral persistence will help to guide therapeutic choices - treatment with small antiviral molecules, for example, may facilitate full clearance of the virus.

Although we are learning much about Ebola from this epidemic, we have yet to identify the events that caused the virus to jump to the Guinean boy almost two years ago. The consumption of bushmeat has been associated with previous epidemics, and some bushmeat species, such as great apes and forest antelopes, are susceptible to high levels of Ebola-virus replication and die from the infection. They are thus best considered as amplifying hosts, rather than the initial reservoir species (Fig. 1). Prime suspects for the reservoir include several species of bat, although a bat source has not been confirmed for this latest epidemic ${ }^{14}$. Indeed, the animal reservoirs of Ebola may be cloaked by sequestration of the virus in much the same way as its persistence in human survivors, waiting for physiological triggers for transmission to unexposed animals of the same species or to amplifying hosts.

Understanding the triggers of Ebola emergence, the persistence of the virus in humans and the infection dynamics in its animal reservoirs is vital not only for the long-term care of survivors of this epidemic, but also for preventing the next one.

Jonathan L. Heeney is in the Laboratory of Viral Zoonotics, University of Cambridge, Cambridge CB3 OES, UK.

e-mail: jlh66@cam.ac.uk

1. Baize, S. et al. N. Engl. J. Med. 371, 1418-1425 (2014).

2. World Health Organization. http://apps.who.int/ ebola/ebola-situation-reports

3. Deen, G. F. et al. N. Engl. J. Med. http://dx.doi. org/10.1056/NEJMoa1511410 (2015). 
4. Mate, S. E. et al. N. Engl. J. Med. (2015). http:// dx.doi.org/10.1056/NEJMoa1509773 (2015)

5. Martini, G. A. Trans. R. Soc. Trop. Med. Hyg. 63, 295-302 (1969).

6. Smith, D. H. et al. Lancet 319, 816-820 (1982).

7. Rodriguez, L. L. et al. J. Infect. Dis. 179 (Suppl. 1), S170-S176 (1999).

8. Baggi, F. M. et al. Eurosurveillance www.eurosurveillance.org/ViewArticle. aspx?Articleld=20983 (2014)
9. Borchert, M. et al. Trop. Med. Int. Health 7, 902-906 (2002)

10.Bausch, D. G. et al. J. Infect. Dis. 196 (Suppl. 2), S142-S147 (2007).

11.Gulland, A. Br. Med. J. 351, h4336 (2015)

12.Clark, D. V et al. Lancet. Infect. Dis. 15, 905-912 (2015).

13. Nursing Stand. 30 (8), 8 (2015).

14.Pigott, D. M. et al. eLife http://dx.doi.org/10.7554/ eLife.04395 (2014)

\section{The Moon's tilt for gold}

The Moon's current orbit is at odds with theories predicting that its early orbit was in Earth's equatorial plane. Simulations now suggest that its orbit was tilted by gravitational interactions with a few large bodies. SEE LETTER P.492

\section{ROBIN CANUP}

$\mathrm{F}$ our and a half billion years ago, a giant impact with Earth is thought to have created an Earth-orbiting disk of debris that coalesced to form the Moon. 'Inelastic' collisions between such debris would dissipate energy and remove relative up-and-down motions, so that the Moon that assembled from these collisions would orbit approximately in Earth's equatorial plane. Yet the Moon's current orbit implies that its initial orbit was substantially inclined relative to Earth's Equator ${ }^{1}$, a troubling contradiction.

On page 492 of this issue, Pahlevan and Morbidelli ${ }^{2}$ identify a compelling and simple solution to this problem - that the Moon's early orbit was gravitationally jostled into a tilted state by close passes of large objects left over from the formation of the inner planets. The existence of a population of these objects could also explain how elements such as iridium, platinum and gold were delivered to Earth's outer layers after the Moon formed $^{3}$.

The Earth-Moon pair is a dynamically coupled system. The Moon's gravity raises tides on Earth, most notably in the oceans, and gravitational interactions between these tides and the Moon is causing Earth's rotation to slow as the Moon's orbit expands. Tidal interactions also reduce the tilt of the Moon's orbit relative to a preferred plane. This would have coincided with Earth's equatorial plane when the early Moon was orbiting close to Earth and transitioned to the plane of Earth's orbit around the Sun as the Moon's orbit expanded. In the absence of other effects, the current $5^{\circ}$ inclination of the Moon's orbit relative to Earth's orbital plane implies an initial $10^{\circ}$ inclination relative to Earth's equatorial plane when the Moon formed ${ }^{1}, 10$ times larger than expected according to theory ${ }^{4}$.

A seemingly unrelated - until now - set of clues about the conditions soon after the
Moon formed emerge from the abundance of precious metals in the Earth. Elements such as platinum and gold are highly siderophile, which means that they have strong chemical affinities for iron. Because Earth formed in a largely molten state, high-density iron would have readily sunk to the planet's centre to form a core, taking highly siderophile elements with it and efficiently removing these from Earth's upper layers. The fact that we find such elements in relatively high abundance in rocks at Earth's surface suggests that they were delivered to the planet after the end of core formation, through a 'late veneer' of material that added about the last $1 \%$ of Earth's mass ${ }^{5}$.

If Earth's late veneer was delivered by a large number of small impactors, the Moon would have received about $1 / 20$ th as many impactors on the basis of its smaller cross section ${ }^{3}$. But lunar siderophile abundances imply that the Moon received much less than that amount. It thus seems probable that Earth's late veneer was delivered by only a few large impactors, each roughly comparable in size to the Moon, because the Moon would have received less than its proportionate share under these circumstances.

Pahlevan and Morbidelli use computational methods (Monte Carlo simulations) to consider the effects of such a population of large, late-accreting background objects on the Moon's early orbit. Their simulations begin with a Moon orbiting in Earth's equatorial plane close to our planet (Fig. 1). With time, the Moon's orbit expands because of tidal interaction with Earth, and is gravitationally perturbed by the background objects until this population is depleted over typically a few tens of millions of years.

Central to the new work is the recognition that each object that ultimately collides with Earth first undergoes many thousands of non-collisional close passes, a portion of which strongly perturb the Moon's orbit. An object approaching the Moon from a random

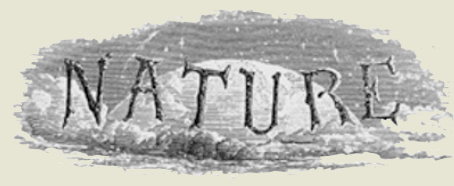

50 Years Ago

The use of rubber gloves during surgical operations became general about 1900 ... The object of an investigation was to obtain an estimation of how frequently wound infection originates from bacteria on the hands of operating staff ... Examination of the wounds following 433 'clean' operations, of the 3,125 rubber gloves used in those operations and of the bacterial flora of the hands which had worn 692 damaged gloves, revealed no connexion between the glove damage, the bacterial flora and the wound infections observed. From Nature 27 November 1965

\section{Years Ago}

The Times of November 20 published a rather flamboyant little article, headed "A Surgical Schism." This article said: "Not for half a century at least has the medical world been so sharply divided as it is to-day in regard to the question of the treatment of wounds." Now, it is exactly half a century since Lister ... first ventured to treat a compound fracture by plugging the wound with a strip of rag soaked in undiluted and impure German creasote. Pyaemia and septicaemia and erysipelas were ravaging the wards of the old Glasgow Infirmary, and he, relying on Pasteur's work on the "germs of putrefaction," and knowing that creasote was a good "disinfectant," plugged a wound with it. That was the beginning of everything, exactly half a century ago. To-day, there are many methods, but they do not all contradict or exclude each other ... We must not imagine a sort of desperate squabble among our military surgeons ... The suggestion in the Times article that an acute controversy is proceeding upon these matters is unfortunate and misleading.

From Nature 25 November 1915 